\title{
Associative Memory design for the Fast TracK processor (FTK) at Atlas
}

A. Annovi, M. Beretta, E. Bossini, F. Crescioli, M. Dell'Orso, P. Giannetti, M. Piendibene, I. Sacco, L. Sartori, R. Tripiccione
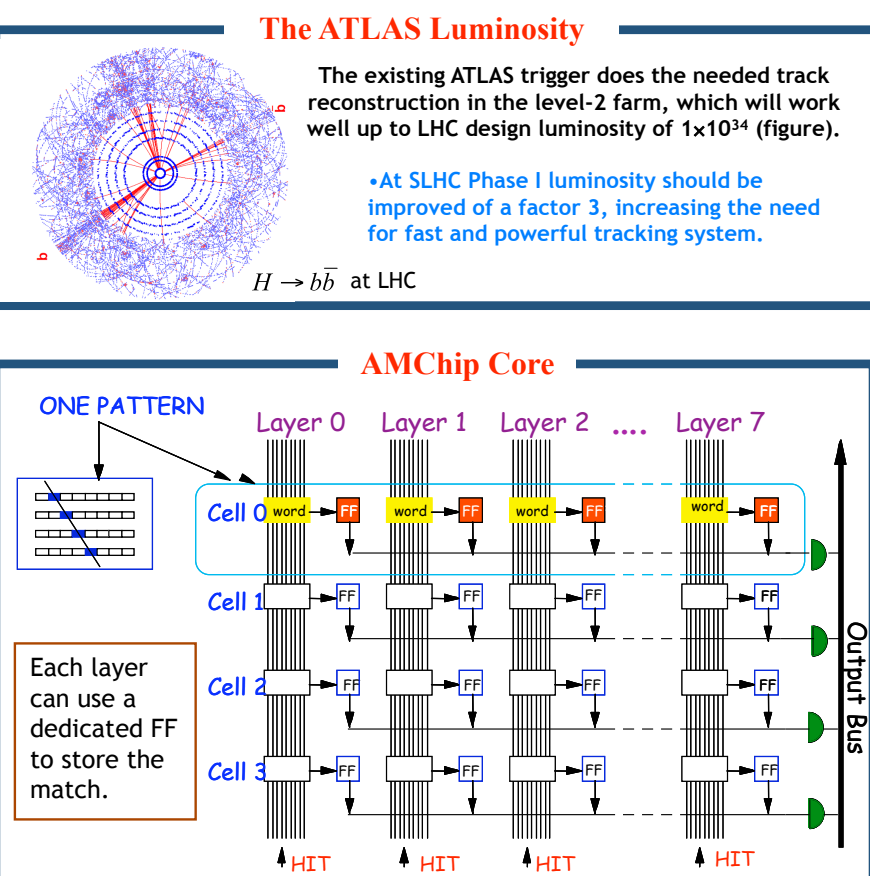

Parallel comparison between the

incoming hit coordinates

with the stored patterns.

Ability to correlate data received at different times.
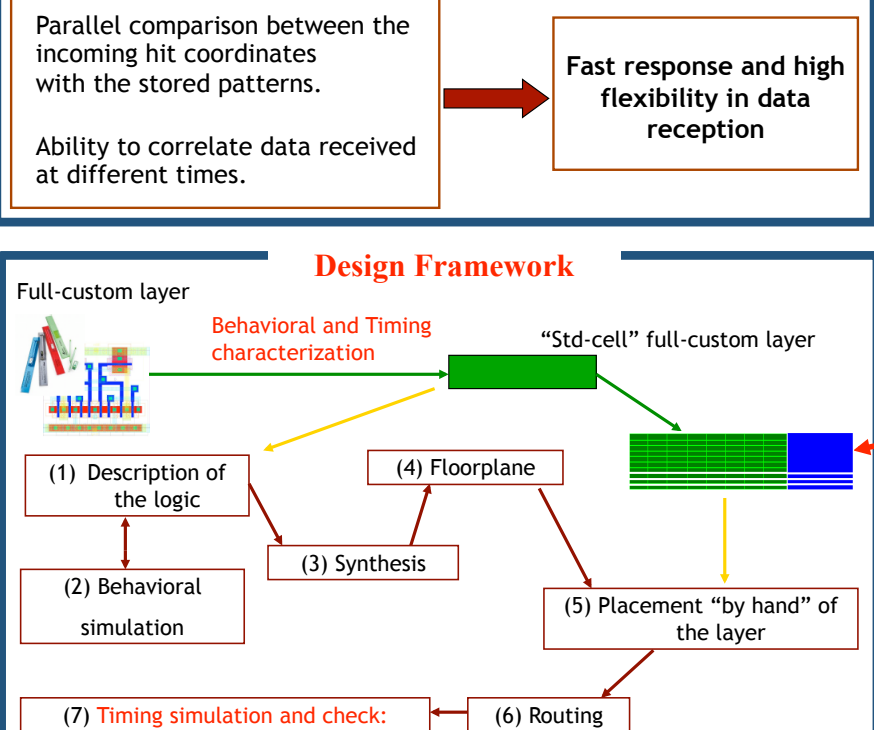

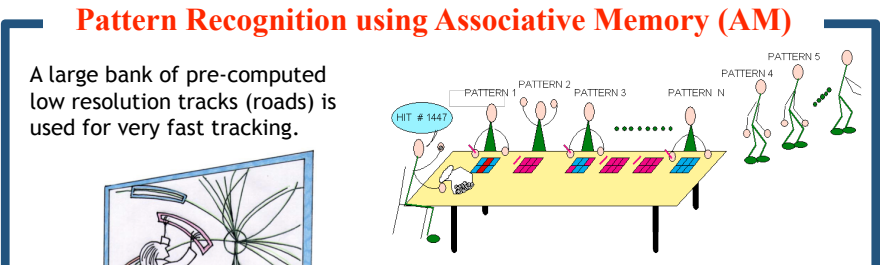

AM [1] finds tracks during detector readout

Full resolution tracks (near offline quality) are extrapolated using fast track techniques inside the roads [2], where linearized fit can be performed.

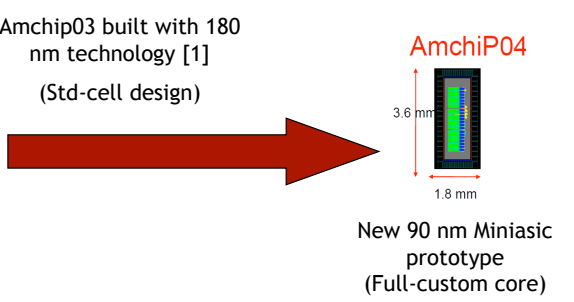

Currently working at

How to improve by a factor 2 pattern density while keeping consumption under control

2. Power saving technique: selective precharge [3]

1.Full custom
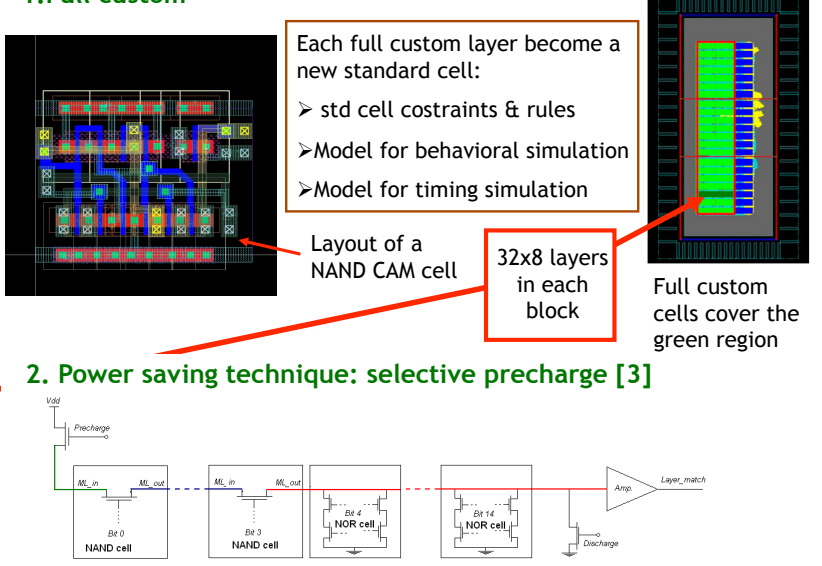

Mixing NOR and NAND cell: the Match-line is precharged ONLY if the first 4 bits have a match. 